\title{
An Experiential Comparative Tool for Board Games
}

\section{Introduction}

As Booth (2015) states, "we are in the midst of a board game renaissance" (p. 2), and with the increased presence of board games in our daily lives comes the increased worth and importance of their analysis as social, textual, and experiential phenomena. This paper aims to present a comparative tool to help compare and differentiate the traits that make board games different, bringing us a step closer to their understanding through their analysis.

In this work, when I refer to a board game, I think of a game that comes packaged as a branded game product, most often in a cardboard box (but may be packaged otherwise), which contains all the physical components that make up that game and are required to play, and is generally designed to be played on a tabletop in one sitting, at the end of which there is one or multiple winners or losers.

The components generally include one or more of the following: boards, cards, cardboard tiles, playing pieces (pawns, meeples, miniatures, cubes, and dice), cardboard tokens, and some other game-specific components (paper money, player screens, player aids, timers, dice-towers, and so on). This comparative tool takes inspiration from a variety of sources. Aarseth et al. (2003) and Elverdam and Aarseth (2007) have inspired the goals of this tool. Elverdam and Aarseth state that a "typology serves best as a tool for comparison" (2007, p. 4), not as a direct solution to the vagueness and unstructured nature of other comparative modalities such as genres. 
Elias, Garfield and Gutschera's systemic analysis (2012, p. 71) was also an inspiration in terms of determining how the model is structured. Similar to Elias et al.'s (2012) systemic analysis, this model will isolate a number of characteristics that affect the general board game experience for the player. These characteristics are each scored separately and objectively to form the board game experience profile of the game. Although Elias et al. (2012) go in depth into no less than 31 different characteristics that could make one game different to the next, it is not presented as an analytical tool which can be practically used for typological analysis, and it is not specific to board games. In this vein, this paper takes inspiration from Elverdam and Aarseth (2007) in creating a tool focused on the ability to compare and highlight differences, with a structural makeup inspired by Elias et al.'s (2012) systemic analysis, as well as an increased focus on usability and practicality.

This model is based on how four different traits of board games generate different experiences for the player. The four traits are as follows:

1. Rules

2. Randomness

3. Representational Backdrop (or Theme)

4. Interaction

Each of the dimensions operates as a continuum; that is, the trait has a minimum and a maximum, and an infinite number of possibilities in-between. For the sake of simplicity and practicality, each of the traits is scored as low, medium, or high, as this is most often sufficient to highlight explicit differences from one game to another. If need be, however, more specific markers along that continuum may be used (such as medium-low, medium-high or 0-10).

\section{Rules}

Before the player is able to play a board game, they need to familiarise themselves with the system. All board games feature some sort of a ruleset. This ruleset will display varying complexities which will affect the player's experience in terms of the time, effort, and investment needed from the player before she is able to play the game. In this model, the effort that the player needs to put in before gaining access to the game is measured through the dimension of rule complexity.

Rule complexity refers to the length, difficulty, and intricacies of the rules. A game like Mage Knight Board Game (Chvátil, 2011) with its two 20-page rulebooks full of intricate rules and rule exceptions cannot be compared to Dixit's (Roubira, 2008) 2-page simple rulebook in terms of rule complexity. Dixit can be explained to experienced 
players and non-experienced players alike in less than five minutes. Mage Knight is known to take hours, if not days, to understand its rules properly.

Mage Knight has high rule complexity, Dixit has low rule complexity. The complexity of the rules will greatly vary the experience of the player, not only in the initial phases of learning, but also in the investment and effort the player will need to maintain throughout the play of the game, for which social situations the game is suitable, and which players are best to play the game with.

The rules on their own do greatly influence experience; however, there is another experiential dimension related to the relationship between the rules and the players. I call this dimension: player reliance.

Many games, especially Eurogames, feature interactions with the rules where the experience of the players mostly involves making choices from a set of available options. This exemplifies a game with low player reliance. Other games, however, greatly rely on the input from the player to be able to function. Some mechanics, such as auctions and trading, although always rule-bound, involve multiple nuanced layers of communication between the players that go above and beyond the rules. In some games, players need to use conversation to be able to build trust in each other and convince the other player that they are on their team, such as in Battlestar Galactica (Konieczka, 2008). Other games, such as Spyfall (Ushan, 2014) and Once Upon a Time (Lambert et al., 1993), require players to make up and use sentences, phrases, stories, and questions around which the experience happens. The generation of this player content is often still rule-regulated; however, the players are using input which has been generated by the players, often regulated by pre-existing social rules, to be able to move gameplay forward.

It is important to differentiate between low player reliance, high player reliance, and Roger Caillois' (1961) concepts of ludus and paidia. Whilst paidia is mostly focused on the absence of rules, player reliance is focused on the need for the player to generate inputs which are outside the game as object. The rules that govern this generation of content, be it trust, a hint, or a story, may seem not to be as strict as the rest of the game's rules; however, this is because the responsibility of regulating these inputs is often transferred to the rules of social construct of the group, instead of the game system itself.

\section{Randomness}

In Dice Games Properly Explained, Knizia (1999) explains the effects of different types of randomness in the clearest and most understandable of formats. He states that the 
different types of games, when it comes to luck, mostly vary with regard to how much control the player has on the outcome of the game. After describing a set of games which he calls "Games of Luck", he states that these games "all have one thing in common: essentially you had no influence over the game. You could hope and cheer for your desired results, but there was little point in pondering about how to play best" (p. 59). He then follows to introduce the next chapters by stating: "This is now going to change. [...] Although the outcome of these games still depends on luck, you have the choice on which bets to place. And that makes a difference" (p. 59, italics in original).

The keyword here is choice. The player's experience shifts considerably by introducing agency (Murray, 1998). As Murray states, agency is "the satisfying power to take meaningful action and see the results of our decisions and choices". Randomness directly affects how meaningful a player's action is within the gamespace, and that directly affects the player's experience of satisfaction. Knizia (1999) describes odds and the "choice of which bets to place" (p. 59) as the factor that is giving the players agency. This choice provider is, of course, specific to the types of games that Knizia proceeds to describe, namely casino dice-based betting games - the main concept being that "more often than not, some bets will offer better returns than others [...] and better chances of winning your games" (p. 59).

This "provider of choice" that Knizia describes translates to many modern board game designs and is a fundamental factor in segregating the two types of luck. Having better odds is simply one of many mechanics that could provide the choice and therefore generate some agency for the player, but this will vary greatly according to the board game mechanisms specific to the game being discussed.

The two main types of randomness, however, remain the same: the randomness that makes decisions for the player, and the randomness that generates options for the player to make decisions on. I call these two types of randomness decisive randomness and productive randomness, respectively.

Greg Costikyan (2008) differentiates between games of skill and games of chan$c e$, stating that very few games are really in those extremes (referring to roulette and chess) and that "almost everything else is some mixture of the two" (Costikyan, 2009, para. 20). Although he does point out that the common dichotomy between skill and chance is a false one, he mostly attributes that falsity to the fact that it is more of a spectrum rather than a binary decision.

Game designer and academic Richard Garfield, in his talk Luck in Games (2011) and later in his book Characteristics of Games (Elias et al., 2012), takes this a step further. He similarly admits that the luck versus skill dichotomy is a false one, but mostly 
attributes this to the fact that they are not even on a spectrum. Essentially, they are not opposites. He argues that there are possibilities of games which factor high skill and also high luck at the same time.

In this classification, randomness is seen as a separate and unrelated factor to skill. The skill required for playing is a resultant of all the different factors discussed in this classification. A game can require skill in that it has high rule complexity and, therefore, a steep learning curve. Another game may require skill congruent to its player reliance in that it requires the player to draw or sing, or potentially memorise a sequence of events, or even be familiar with a specific universe. Highly player reliant games often also require skills of social interaction and emotional intelligence, where agreements need to be made between players and the ability to convince other players is crucial in winning the game. Randomness on its own is still regarded as a continuum. A game can vary from a high dependence on randomness to no randomness whatsoever.

Let us look at a few examples of types of randomness, starting off with the classic roll and move mechanic. The player rolls a six-sided die and then moves the pawn or playing piece that number of steps on the board. This is an example of decisive randomness through overt luck. The dice make a decision for the player with regard to how many spaces he/she can move. The player has no possibility to influence that die roll, and even if he/she does, that would not change the fact that the dice make the decision, which is final, onto where the player will be able to move. This could be mitigated through the use of a board. For example, in Snakes and Ladders, the track is linear - therefore, the decision of the die immediately dictates the exact space to which the player will be able to move.

Let us imagine a hypothetical move mechanic where the player, instead of rolling 1 die, rolls 3 dice, and the player then gets to choose which of the three dice he/she would like to use for his/her move. This is a case of productive randomness. The dice generate a set of three options for the player, and the player makes a choice, selecting the best out of a set of available options to best suit her current game state.

The difference here is that the dice's decision in Snakes and Ladders is unique and final, while in the above example of productive luck, the dice create further choices for the player, instead of making a decision for the player.

These two types of luck can also be used in different combinations of each other in a single game. It is true that decisive luck is generally regarded within the game design community as "bad randomness"; however, designers have managed to use it in different ways to either conceal it or use it to create an experience where players still feel that they are able to exert their agency in the game. 
Decisive randomness is a common feature in many modern board games, even Eurogames. In modern board games, however, these mechanics rarely feature without some sort of mitigation (Knizia, 1999). If they are unmitigated, they are generally used for a global effect, affecting all of the players equally without a specific advantage (or disadvantage, for that matter) to any single player. The popular board game The Settlers of Catan (Teuber, 1995) features unmitigated decisive randomness in deciding which resources are produced each turn. The 2014 Kennerspiel des Jahres winner Istanbul (Dorn, 2014) features unmitigated decisive randomness in selecting where certain non-player controlled characters appear and move to on the board. The adventure game Mage Knight Board Game features partly-mitigated decisive randomness in the random drawing of a monster, and unmitigated decisive randomness in the use of the "dummy player" mechanic when playing with less than four players.

\section{The Representational Backdrop}

The game's theme is a selling factor in most games. When one describes a game to another person a short description of the theme often ensues: "you travel with camels across the desert" or "you build spaceships and then watch them as they are broken apart by meteors". A game tends to capture your attention because it sounds compelling, simply through the theme. Most games have a theme; how relevant that theme is to the actual game mechanics is another question altogether. The theme is sometimes used as the basis of the game, creating mechanics that attempt to evoke the narrative or emotions that the theme affords. In other situations, the theme simply works as a container, to act as a context to the art and the game mechanics, which may or may not have been developed with a theme in mind. This section, therefore, presents the dimension of theme syner$g y$, which measures the extent of synergy found between the game's theme, aesthetics, and mechanics. "Theme" represents the setting or "what you are doing in the game". Aesthetics is a combination of the visual and physical elements of the game. This mostly includes the art, the representative elements (the cards, the board), and any miniatures or playing pieces. Mechanics represent the mechanical function of the game's play.

A commonly-used phrase within the board game community is that of "pasted-on theme", referring to a theme which was clearly added in the later stages of the development and is pretty much inconsequential to gameplay. For some other players or designers, theme is important, as they may enjoy the narrative elements of the game more than the interaction with the system.

This extent of theme synergy is useful in describing to the player where the focus of the game experience lies. A game where the theme is central to its gameplay will inform 
a player that he/she expects to play a game where he/she should be able to play the role, create a story and play the game with the theme in mind. A game with a low theme synergy would create an experience more based around the ludic aspects with specific attention to the mechanics, and how to best make use of them to outsmart an opponent.

Some games, especially paratextual board games, rely on their high theme synergy, as a selling point but also in form and function. Booth (2015) discusses a variety of paratexutal board games and the relationship between the players, and the characters that have been translated from other media. He discusses how paratextual board games and the relevance of the mechanics to the theme in general are capable of opening player dialogue with the media text. Booth also states: "the materiality of the game pieces in paratextual board games facilitates fan interaction with the game as a system while also externalizing the game as an additional episode within the media franchise" (2015, p. 17).

Games with high theme synergy will feature mechanics specifically intended to convey the theme upon which they set out to be based. A game about killing monsters will most certainly involve combat, weapons, characters, and mechanics to afford its own theme. Greg Costikyan (2011) describes Ameritrash games by means of a trait similar to theme synergy, where he states that they focus on a "tight connection between theme and mechanics" (Costikyan \& Davidson, 2011, p. 183). A game with high theme synergy is therefore one where, if it were to be stripped from its theme, the experience is significantly adversely altered to an extent that mechanics lose context and imbued meaning, to a point of potential redundancy. Fighting heroes becomes a matter of who rolls higher. Exploration becomes a matter of flipping a tile or card, and hoping for the symbols that you would have liked. Because flipping a tile was a mechanic that was meant to inspire a feeling of exploration, when any connection to exploration is removed from the equation, the generated experience runs the risk of being underwhelming. A great focus on specific representative game pieces and aesthetics, such as miniatures, maps and locations, as well as a good amount of effort in contextualizing specific mechanics as an attempt to simulate meaningful actions within the context of the theme, are often good indicators of high theme synergy. These games often contain rules that are there specifically to evoke the theme and tend to feel less natural or obvious when observed objectively without the contextualization and framing as provided by theme.

Games with medium theme synergy are ones where the relationship between theme and mechanics is still very significant, and removal of theme would have some adverse effects, but not to an extent that specific rules or mechanics appear to be redundant. 
In simpler terms, in games with high theme synergy, the rules and mechanics simulate and evoke the theme, while in games with medium theme synergy, the theme complements and elevates the mechanics.

Not all games need to be based on a theme. Not all games have a theme at all. Games with low theme reliance are games where the theme, present or not, is not the main event in terms of the game's appeal and gameplay. The mechanics are not based or tied to a specific event or sequence thereof. From a commercial perspective, they are often still themed, mostly to allow some art to be made, but the game would stand on its own right without the theme. This is more commonly seen in Eurogames, and is also reflected in the aesthetics. While games with high theme synergy are generally accompanied by highly iconic playing pieces - such as numerous plastic miniatures to fit the game world, Eurogames often feature more symbolic wooden pieces, such as pawns or meeples, as although the theme is important, the designer or the publisher would prefer to highlight the mechanical properties. When a game has a less strongly represented theme, less representative pieces and a lessened amount of mechanics directed towards evoking a certain theme, the game is regarded as low theme synergy. In other words, a game with low theme synergy is one where, if it were to be stripped of its theme, the experience would remain mostly unchanged, with little or no adverse effects.

Completely abstract games with no theme whatsoever, such as Haggis (Ross, 2010), are scored as having no theme synergy. Theme synergy is one continuum that can frequently be scored as nil.

\section{Interaction}

Game designer Lewis Pulsipher talks about the "petty diplomacy" problem (2011). This is a specific situation where, in three-player competitive games with interaction, the leader would always be beat down by the other two players to prevent her from winning, resulting in a never-ending stalemate. He mentions that "this is not usually a problem in games where players can do little to affect other players, such as most race games" (Pulsipher, in Costikyan \& Davidson, 2011, p. 17). He continues to state that "many "Euro"-style board and card games (which are often for three or four players) have been called "multiplayer solitaire", a popular style partly because it avoids the "petty diplomacy" problem" (p. 17).

The term "multiplayer solitaire" refers to a subset of games where players have very little possibility to interact with each other from a game perspective, leaving them essentially playing a game on their own, with little need to look at other players' game states. When the game ends, the players often compare points to see who wins the game. Social interaction while playing may still be present, but not interaction through the game itself. 
The extent of player interaction is a factor which causes much debate within the board game community. This is evident in an article on the game criticism website and show Shut up and Sit Down (Smith \& Dean, 2011). The two reviewers argue on the value of multiplayer solitaire and interaction in board games, one of them stating:

Games where, fundamentally, each player is off in their own world, worrying about their own problems, and not in anything resembling dynamic conflict or co-operation with their friends. I find them dull. But I'd go further than that. I'd actually call them failures of design. (Smith \& Dean, 2011, para. 3)

In their arguments, they classify the popular board game Agricola (Rosenberg, 2007) as multiplayer solitaire. While one reviewer and game critic classifies it a "failure in design", the game has maintained its position in the top ten board games on boardgamegeek for more than half a decade. In fact, if one had to look at the list of the top ten games rated by the worldwide community, five of the top ten have strong elements of multiplayer solitaire. The above quote also introduces two other types of interaction that the said critic finds enjoyable, namely dynamic conflict and co-operation.

Smith points out specific and significantly different game experiences from one game to the other, based on how much interaction between players the game affords. Whether a game is that of competition or of collaboration is a simple and functional differentiator. Competition and collaboration, similar to luck and skill, are another example of a false dichotomy. Although the two may seem opposite at first, one does in fact allow for another in the course as well as the flow of a single game. A straightforward example would be that of the board game Diplomacy (Calhamer, 1959), where a collaborative ally eventually becomes a backstabbing rival. This does not mean that Diplomacy is not a competitive game. It simply means that although it is, in essence, a competitive game, it affords some sort of co-operation.

The same applies for two-team games such as Tichu (Hostettler, 1991), where the team-mates collaborate with each other to compete against the opposing team, therefore featuring high levels of co-operation as well as competition. Therefore, similar to many of the other factors in this classification, player interaction works on two separate independent spectra: collaborative interaction and competitive interaction.

A game with high competitive interaction and no collaborative interaction would be one where there is a two-player conflict, such as a simulation of a war. For example, the Commands and Colors series (Borg, 2006). The two players are in a constant competition against each other and each move is premeditated so as to benefit the player or 
damage the opponent, although the players most often attempt to achieve both at the same time. As soon as a third player is introduced, a potential element of co-operation emerges (as in the previously-discussed situation of petty diplomacy) and, therefore, the game is no longer solely based on competitive interactions.

A game with medium competitive interaction and no co-operative interaction is that of the classic Euro-style game. The players have little opportunities for direct attacks; however, they have multiple opportunities to rush ahead of their opponents, mixed in with a few opportunities to stall the opponent's progress. If a trading mechanic is introduced, this immediately increases the level of co-operative interaction, as "fair" trades are almost always symbiotic.

A game with high co-operative interaction and low competitive interaction would be that of the semi-cooperative game, such as Archipelago (Boelinger, 2012) and CO2 (Lacerda, 2012). The players play almost the entire game in a co-operative fashion; in most cases, attempting to prevent a specific game condition that would make the players lose collectively. Once the game ends, however, only one player wins the game. The competition becomes understated and almost secretive. In fact, most of these games tend to feature secret winning objectives for each player which are only revealed at the end.

A game with only collaborative interaction would be a fully cooperative game with no traitor mechanic, such as Pandemic (Leacock, 2007) and ... and then we held hands (Chircop \& Massa, 2015). These games' only outcomes are that either everybody wins, or everybody loses. All the interactions in such games are towards the collective win.

Depending on the type of game that is being played, interaction will play a more or less significant role. Whatever the case, it should be noted that apart from the interactions that happen between the players' playing pieces, there is an overlying layer of social interaction which the game generates that is present regardless of which type of interaction it exhibits.

\section{Using the Tool}

This tool is intended to be a comparative tool, that is, useful mostly to point out differences between two or more board games. When classifying different styles of games, one will notice that similarities and differences will become apparent, and patterns that define a certain experience will emerge.

In this section, I will briefly outline the use of the model and display some examples of games as classified by it. To classify a game, the user should go through all of the seven differentiators and score the game as low, medium, or high. In some cases, although quite rare, some games could have one or more of the traits classified as nil. Although 
this could occur, it is more common for games to have many of the traits, even if they are classified as low. Pandemic (Leacock, 2007), for instance, is one game which features no competition between players whatsoever and, for this reason, scores a nil on competitive interaction. It should be noted that a score of nil is still a score, and should not be omitted from the classification. The typology becomes particularly useful when displayed in a bar-graph format, as a visual representation of the traits creates a clear visual distinction from one game to another and allows for visible patterns to emerge.

An interesting starting point would be to compare two games from the two overarching genres of board games - Ameritrash and Eurogames.

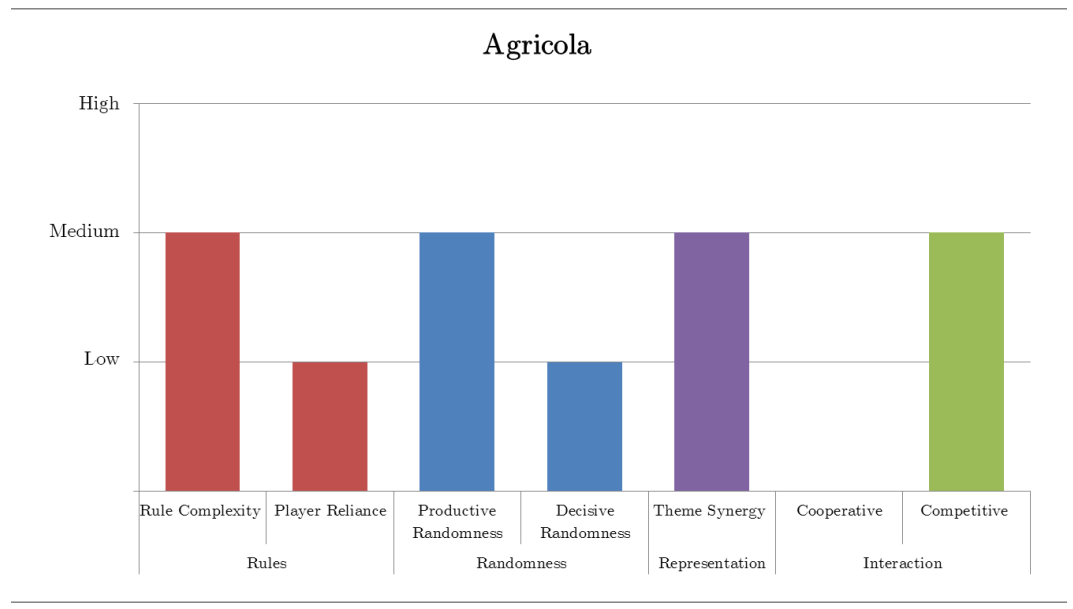

Figure 1. Board game experience profile for Agricola

Agricola (Rosenberg, 2007) and Descent: Journeys in the Dark (Wilson, 2005) offer two very different experiences. Agricola (see Figure 1), as a Eurogame, seems to offer a relatively balanced experience with no high extremes. The scores seem to be close to each other, with more than half the traits scored on medium. There is no single trait that is outstanding, indicating a multi-faceted experience of some competition, thoughtful gameplay and representation, with a moderated dose of productive randomness to keep things fresh from game to game. This moderated game design is a common trait in many Eurogames, as we shall see when we compare two other types of games.

Descent (see Figure 2), on the other hand, has more than half the traits scored as high, and a tendency to the extremes. The game has high scores for randomness (especially the decisive variety), with very high interaction between players both on the cooperative as well as the competitive dimensions. The game has higher theme synergy 
than the Eurogame, which is a common trait in Ameritrash-style games. Ameritrash games therefore tend to have traits that generally score higher in the extremes and are generally skewed to the right (theme and interaction).

Descent

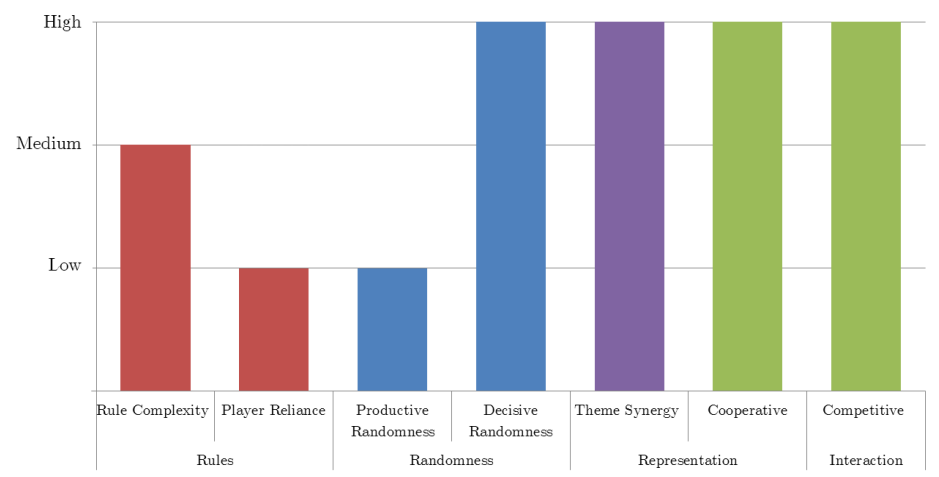

Figure 2. Board game experience profile for Descent

The Castles of Burgundy

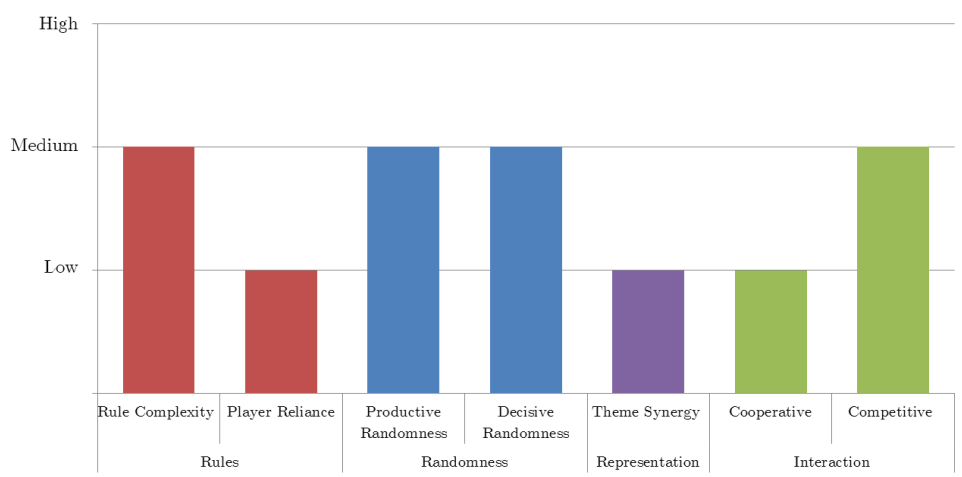

Figure 3. Board game experience profile for The Castles of Burgundy

The model is not necessarily only used to compare functionally-opposite board games, but can also be used to isolate experiential differences between similar games.

Agricola and The Castles of Burgundy (Feld, 2011) (see Figure 3) are both Eurogames that are well-respected in the board game community. Although one could still fit both of them within the Eurogame category, they do feature slightly different experiences. As one can see, The Castles of Burgundy still features the moderation that 
we saw in Agricola; however, it has less thematic synergy and a greater feature of randomness. This is a product of the use of dice and the use of less representative pieces.

If we look at a completely different style of game, such as the more mass market games, we will notice that the profile will not explicitly feature any of the characteristics or patterns that emerged in Eurogames and Ameritrash games.

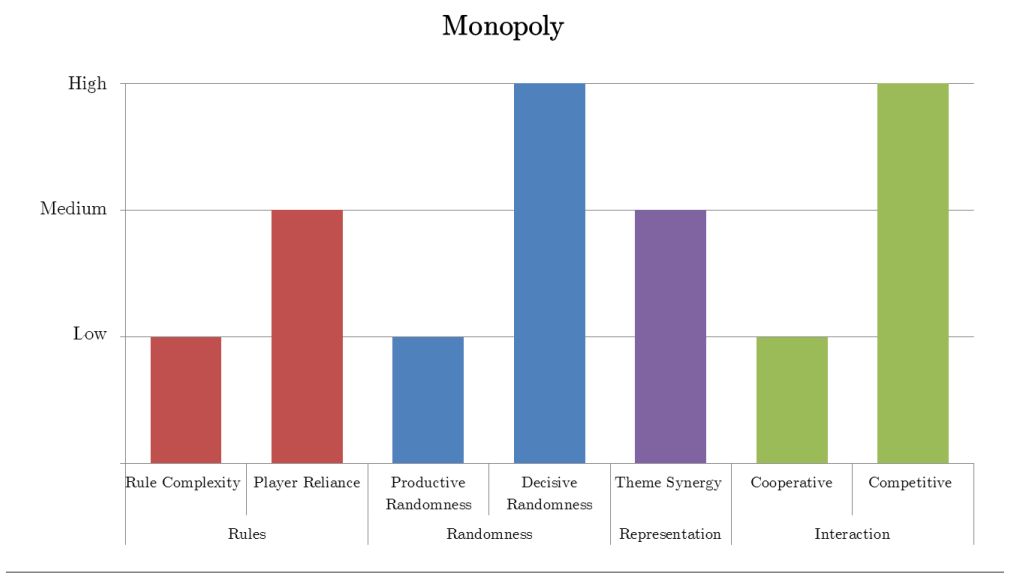

Figure 4. Board game experience profile of Monopoly

One would notice that this group of games features a lot less variation from this common formula than the other types of games. As can be seen in Figure 4, the games often feature relatively simple mechanics (roll and move) combined with an overlying loosely player-reliant mechanic. For instance, in Monopoly (Darrow \& Magie, 1935) (see Figure 4), there is trading as a loose overlay; in Clue (Pratt, 1949), the roll-and-move mechanic is overlaid with a deduction mechanic. The games are almost exclusively competitive, with little cooperation. The traits that are found in early mass market games are still featured in some more recent designs; for instance, the game Exploding Kittens (Inman, Lee, \& Small, 2015) features a very similar experience profile to Monopoly, which could explain why the game was well-received by the mass market, raising millions of dollars on Kickstarter, but received a mixed reaction from the more critical and analytical boardgamegeek community.

Another notable experience is that of more social, player-reliant games, such as The Resistance (Eskridge, 2009), see Figure 5. 


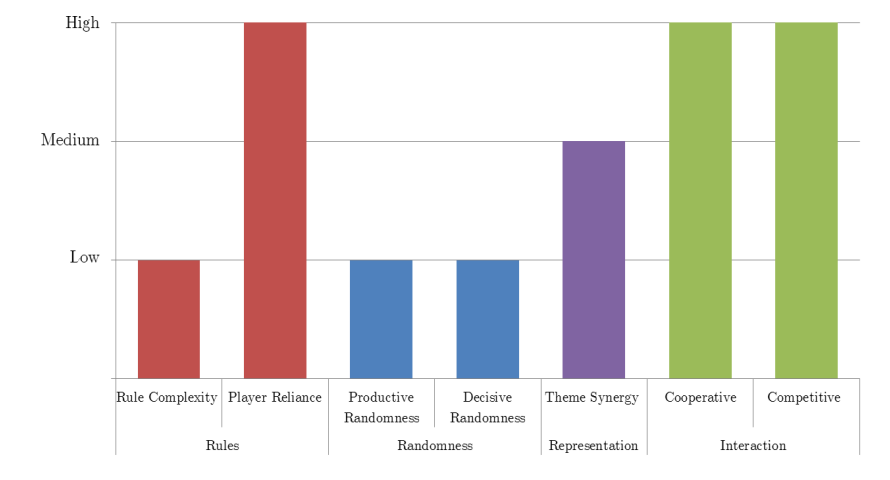

Figure 5. Board game experience profile for The Resistance

Here we see an example of game design where there is high priority on extensive interaction. This is reflected by the high occurrence of player-oriented traits; that is, player reliance and interaction, and then a low occurrence on most other traits. This experience is geared towards the social aspects of gameplay and gives the more ludic aspects, such as rules and types of randomness, a much lesser value. This displays a "specialist" profile pattern, in that the game seeks to specialise in some specific traits and highlight them.

Another contrasting profile pattern is that which I call the "maximalist" design pattern. Here the game seems to fully maximise all the traits that it incorporates. An example of this is Mage Knight Board Game, a game that aims to be highly thematic, but also highly strategic, giving as much control to the player as possible. This is evidently reflected in the game's experience profile (see Figure 6).

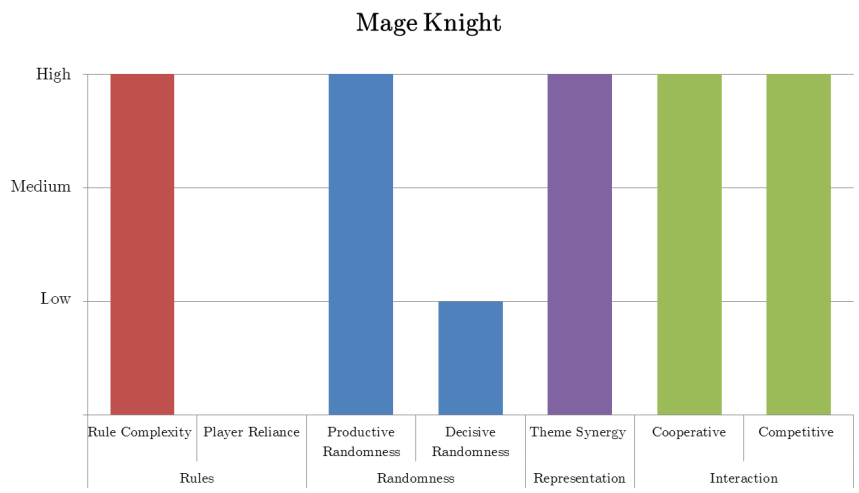

Figure 6. Board game experience profile for Mage Knight Board Game 
Although implications as a descriptive and comparative tool have been clearly demonstrated in this paper, there are some potential applications as a game design tool that are worth outlining. Perhaps the most practical of its uses lies in its ability to give the designer an alternate perspective focused on player experience, rather than specific mechanics or genres. This applies both proactively, when drafting and visualizing the desired player experience for a prototype, but also retroactively, when analyzing actual player response compared to the expected one. Due to its simple nature and its focus on player experience, the tool has potential applications for data collection, where the players could plot their experience of a prototype using the tool, which could then be compared to the designer's vision of it, visually revealing discrepancies between the player's current experience and the designer's desired effect.

\section{Conclusion}

The comparative tool presented in this work, albeit within itself relatively basic, is the first step into the deeper understanding of the board game medium. It is the understanding of subtle differences between the different experiences the medium is capable of generating that will lead us to a greater understanding of its overall cultural significance. Although the above discussed observations are derived from the author's subjective analysis, this paper has showcased the capability of this tool to be able to display diverse combinations of its basic traits and has already allowed to witness a development of how the medium itself changed over the last few decades. Influences and trends could be displayed visually, and evident patterns emerged from the analysis of a few key games within the medium and the different focuses that different designers choose to employ. This comparative tool is a first step towards a much needed thorough typology specifically designed for board games while also providing a framework and direction for future empirical research on the subject, without sidelining the medium's cultural and social circumstances.

\section{References}

Aarseth, E., Smedstad, S., \& Sunnanå, L. (2003). A Multi-Dimensional Typology of Games. Utrecht: DiGRA.

Boardgamegeek.com. (2015). BoardGameGeek | Gaming Unplugged Since 2000. Retrieved 1 July 2015, from http://www.boardgamegeek.com

Booth, P. (2015). Game Play: Paratextuality in Contemporary Board Games. New York: Bloomsbury. 
Caillois, R. (1961). Man, Play and Games. Urbana and Chicago: Univeristy of Illinois Press.

Calleja, G. (2011). In-Game. Cambridge, MA: The MIT Press.

Costikyan, G. (2008). Uncertainty in Games. Cambridge, MA: The MIT Press.

Costikyan, G., \& Davidson, D. (2011). Tabletop. Pittsburgh, PA: ETC Press.

Elias, G., Garfield, R., \& Gutschera, K. (2012). Characteristics of Games. Cambridge, MA: MIT Press.

Elverdam, C., \& Aarseth, E. (2007). Game Classification and Game Design: Construction Through Critical Analysis. Games and Culture, 2(1), 3-22. doi:10.1177/1555412006286892

Garfield, R. (2011). Luck in Games. Presentation, NYU Game Series.

Knizia, R. (1999). Dice Games Properly Explained. Tadworth, Surrey: Right Way.

Murray, J. H. (1998). Hamlet on the Holodeck: The Future of Narrative in Cyberspace. Cambridge, MA: MIT Press.

Parlett, D. (1999). The Oxford History of Board Games. Oxford: Oxford University Press.

Smith, Q., \& Dean, P. (2011). It's War: Player Interaction. Shut Up \& Sit Down. Retrieved 1 July 2015, from http://www.shutupandsitdown.com/blog/post/its-warplayer-interaction/

Woods, S. (2012). Eurogames. Jefferson, N.C.: McFarland \& Co.

Ludography

Boelinger, Ch. (2012). Archipelago. [Board Game], Asmodee.

Borg, R. (2006). Commands and Colors: Ancients. [Board Game], GMT Games.

Calhamer, A. B. (1959). Diplomacy. [Board Game], Avalon Hill.

Chircop, D. \& Massa, Y. (2015). ... and then, we held hands. [Board Game], LudiCreations.

Chvátil, V. (2007). Galaxy Trucker. [Board Game], Czech Games Edition.

Chvátil, V. (2011). Mage Knight Board Game. [Board Game], WizKids Games.

Darrow, C., Maggie. (1935). Monopoly. [Board Game], Parker Brothers.

Dorn, R. (2014). Istanbul. [Board Game], Pegasus Spiele.

Eskridge, D. (2009). The Resistance. [Board Game], Indie Boards and Cards.

Feld, S. (2011). The Castles of Burgundy. [Board Game], Alea.

Hostettler, U. (1991). Tichu. [Board Game], ABACUSSPIELE. 
Inman, M., Lee, E., \& Small, Sh. (2015). Exploding Kittens. [Board Game], self-published.

Knizia, R. (1998). Through the Desert. [Board Game], Fantasy Flight Games.

Konieczka, C. (2008). Battlestar Galactica. [Board Game], Fantasy Flight Games.

Lacerda, V. (2012). CO2. [Board Game], Giochix.it.

Lambert, R., Rilstone, A., Wallis, J. (1993). Once Upon a Time: The Storytelling Card

Game. [Board Game], Atlas Games.

Leacock, M. (2007). Pandemic. [Board Game], Z-Man Games.

Pratt, A. (1949). Clue. [Board Game], Leeds, Unknown.

Rosenberg, U. (2007). Agricola. [Board Game], Lookout Games.

Ross, S. (2010). Haggis. [Board Game], Indie Boards and Cards.

Roubira, J.-L. (2008). Dixit. [Board Game], Libellund.

Teuber, C. (1995). Die Siedler von Catan. [Board Game], KOSMOS.

Ushan, A. (2014). Spyfall. [Board Game], Cryptozoic Entertainment.

Wilson, K. (2005). Descent: Journeys in the Dark. [Board Game], Fantasy Flight Games.

\section{Summary}

In the field of game studies, contemporary board games have until now remained relatively unexplored. The recent years have allowed us to witness the emergence of the occasional academic texts focusing on board games - such as Eurogames (Woods, 2012), Characteristics of Games (Elias et al. 2013), and most recently Game Play: Paratextuality in Contemporary Board Games (Booth, 2015). The mentioned authors all explore board games from diverse viewpoints but none of these authors present a viable and practical analytical tool to allow us to examine and differentiate one board game from another. In this vein, this paper seeks to present an analytical comparative tool intended specifically for board games. The tool builds upon previous works (Aarseth et al. 2003; Elias et al. 2012; and Woods 2012) to show how four categories - rules, luck, interaction and theme - can interact on different levels to generate diverse gameplay experiences. Such a tool allows to score games objectively and separately in each of the categories to create a combined gameplay experience profile for each board game. Following this, the paper proceeds to present numerous practical examples of contemporary board games and how it can be used from a design perspective and an analytical perspective alike. 
Keywords: board games, analysis, comparison, player experience, typology

David Chircop is a game designer from Malta. He was involved in the international publication of more than 10 board games in roles such as Designer, Developer, Project Lead and Publisher. His master's thesis focused on building a model for the analysis of player experience in contemporary board games. Currently, David works as a Game Designer at CD Projekt RED. 